Pacific Journal of Mathematics

TREES AND PROTO-METRIZABLE SPACES 


\title{
TREES AND PROTO-METRIZABLE SPACES
}

\author{
LUTHER B. FULLER
}

It is known that metrizable spaces are characterized as the compact closed continuous image of a subspace of some Baire zero-dimensional space and that compact metrizable spaces are characterized as the closed continuous image of the Cantor set.

In this paper we investigate some of the properties of trees and non-archemedian spaces and provide, among others, a characterization of proto-metrizable spaces which generalizes the above characterizations of metrizable spaces by showing that a proto-metrizable space is the image of a non-archemedian space under an (irreducible) closed map such that each point pre-image is either a point or a compact $G_{\delta}$-set. This result specializes a characterization of paracompact spaces as the image under a compact closed map of an ultra-paracompact space.

We then show that non-archemedian spaces and their irreducible closed continuous images have a normality property, called $M_{1}$-normality, and it follows that proto-metrizable spaces are $M_{1}$-normal.

I. Introduction. The characterizations of paracompact and compact metrizable spaces mentioned above are due, respectively, to Ponomarev [14] and to Aleksandrov and Uryson [1]. The characterization of metrizable spaces may be found in [9, p. 218]. Proto-metrizable spaces were introduced by Nyikos [12] as a generalization of non-archemedian and metrizable spaces. Extensive background material on non-archemedian spaces has been provided by Nyikos [10], [11], [12], [13] and will not be covered here.

Recently, Gruenhage and Zenor [3] have characterized proto-metrizable spaces as spaces having a rank-1 pair-base. A rank-1 base [2] is a base such that if two elements intersect, then one is a subset of the other. A pair-base is a collection of pairs $(b(1), b(2))$ of sets such that $b(1)$ is open, $b(1) \subset b(2)$ and if the open set $u$ contains $x$, then there exists a pair $(b(1), b(2))$ with $x \in b(1) \subset b(2) \subset u$. A rank-1 pair-base is a pair-base such that if $(b(1), b(2))$ and $\left(b^{\prime}(1), b^{\prime}(2)\right)$ are elements with $b(1) \cap b^{\prime}(1) \neq$ 0 , then either $b(1) \subset b^{\prime}(2)$ or $b^{\prime}(1) \subset b(2)$.

The set-theoretic notation used here is that of Jech $[5, \mathrm{p} .1]$ with the addition that we use $\mathcal{E}$ as the classifier instead of the more usual braces (except when the elements of a set are listed, e.g. $\{x\}$ ). For example, "the set of all $x$ such that $x$ has property $P$ " is denoted $\mathcal{E} x(x$ has property $P)$. All other operations such as union, intersection, lub, etc., are assumed to incorporate the classifier, thus, for example, "the least upper bound of the set of all $x$ having property $P$ " is denoted lub $x$ ( $x$ has property $P)$. 
Ordinals are assumed to be transitive sets well-ordered by $\in$, so we denote " $\alpha$ is less than $\beta$ " by $\alpha \in \beta$. See [5, p. 7]. The empty set is denoted by the ordinal 0 , the set of finite ordinals is $\omega$ and $\omega(\alpha)$ is the $\alpha$-th infinite cardinal.

The operations "closure" and "interior" are denoted $\mathrm{Cl}$ and In respectively and the space in which these operations are carried out is denoted by a subscript where necessary for clarity.

The "star" of a collection and $\Delta$-refinement are frequently used $[9, \mathrm{p}$. 50]: If $U$ and $V$ are covers of $X$, then $V$ is a $\Delta$-refinement of $U$, denoted $U \ll V$, if for each $x \in X$ there exists $u \in U$ such that $\operatorname{St}(x, V)=$ $\cup v(x \in v$ and $v \in V) \subset u$.

II. Proto-uniformizing families and ortho-bases. A proto-uniformizing family for a space $X$ is a family $\mathcal{Q}=\varepsilon U(\alpha)(\alpha \in \gamma)$ of collections of open subsets of $X$ such that:

(1) $\cup U(\alpha)(\alpha \in \gamma)$ is a base for $X$; and

(2) for each $\alpha \in \gamma$ there exists $\beta \in \gamma$ such that $\cup U(\alpha)=\cup U(\beta)$ and $U(\beta) \Delta$-refines $U(\alpha)$.

$\mathcal{Q}$ is said to be well-ordered by refinement if, in addition, for each $\alpha$, $\beta \in \gamma, \alpha \in \beta$ implies that $U(\beta) \Delta$-refines $U(\alpha)$.

A base $B$ for a space is called an ortho-base if whenever $B^{\prime} \subset B$ and $x \in \cap B^{\prime}$, then either $\cap B^{\prime}$ is open or $B^{\prime}$ contains a base at $x$. A space is proto-metrizable if it is paracompact and has an ortho-base. It is known [12, Theorem 4.3] that a space is proto-metrizable if and only if it has a proto-uniformizing family well-ordered by refinement.

A collection of sets is said to be totally ordered if its ordering by set inclusion is a total ordering. A sequence of open sets is said to be perfectly decreasing if it is well-ordered by reverse set inclusion so that the closure of each element is a proper subset of each of the preceding elements.

A base $B$ for a space $X$ is called a (perfectly) monotone ortho-base if whenever $B^{\prime}$ is a totally ordered (perfectly decreasing) subset of $B$ and $\cap B^{\prime}$ contains $x$, then either $\cap B^{\prime}$ is open or $B^{\prime}$ contains a base at $x$.

A set is said to be canonical if it contains the interior of its closure and it is a subset of the closure its interior.

If $U$ is a collection of subsets of a space, then $U$ is said to be locally finite-in-itself if for each $x \in \cup U, U$ is locally finite at $x$. Denote by $\mathcal{L}(X)$ the set of all collections of canonical open subsets of the space $X$ which are locally finite-in-themselves.

Let $B$ be a base for $X$ and let $<B$ denote the order on $\mathcal{L}(X)$ such that for each $U, V \in \mathcal{L}(X), U<B V$ if and only if for each $v \in V$ there exists $b \in B$ and $u \in U$ such that $V \subset b \subset \mathrm{Cl} b \subset \neq u$ unless $u$ is an isolated point, in which case $v=b=\mathrm{Cl} b=u$. Notice that the ordering $\angle B$ does not require that $\cup V=\cup U$ whenever $U<B V$. 
LEMMA 1. If $X$ is a paracompact space and $B$ is a base for $X$, then for each $U \in \mathcal{L}(X)$ which covers $X$ there exists $V \in \mathcal{L}(X)$ such that $\cup U=$ $\cup V$ and $U \ll B V$.

Proof. Suppose $U \in \mathcal{E}(X)$ is a cover of $X$. Since $X$ is regular, for each $x \in X$, there exists $u \in U$ and $b(x) \in B$ such that $x \in b(x) \subset \mathrm{Cl} b(x)$ $\subset \neq u$ unless the only element of $U$ containing $x$ is $\{x\}$ in which case we have $x \in\{x\}=b(x)=u$.

Let $V(3)=\mathscr{E} b(x)(x \in X)$ and in the same manner construct $V(2) \subset$ $B$ from $V(3) . V(2)$ is an open cover of $X$ and since $X$ is paracompact, $V(2)$ has a locally finite $\Delta$-refinement $V(1)$ covering $X$.

Let $V=\mathcal{E} \operatorname{InCl} v(v \in V(1))$, then $V \in \mathcal{L}(X)$. In order to show that $U \ll B V$ we observe that for each $x \in X$ there exists $v(3) \in V(3), v(2) \in$ $V(2)$ and $u \in U$ such that either $\operatorname{St}(x, V) \subset \mathrm{ClSt}(x, V(1)) \subset \mathrm{Cl} v(2) \subset \neq$ $v(3) \subset \mathrm{Cl} v(3) \subset \neq u$ or $\operatorname{St}(x, V)=\{x\}=v(3)=\mathrm{Cl} v(3)=u$.

LEMMA 2. Suppose $X$ admits a perfectly monotone ortho-base $B$ and $\mathscr{Q}=$ $\mathcal{E} U(\alpha)(\alpha \in \gamma) \subset \mathcal{L}(X)$ with the following properties:

(a) $\gamma$ is a limit ordinal and for each $\alpha, \beta \in \gamma, \alpha \in \beta$ implies $U(\alpha)$ $\ll B U(\beta)$;

(b) for each $\alpha \in \gamma$ and $x \in X$, either $x \in \cup U(\alpha)$ or $\cup U(\beta)(\beta \in \alpha)$ contains a base at $x$; and

(c) for each $\alpha \in \gamma, \cup U(\alpha)=\cup U(\alpha+1)$; then there exists $U(\gamma) \in \mathcal{L}(X)$ such that:

(1) $U(\gamma)$ is a pair-wise disjoint collection of closed-open sets;

(2) for each $\alpha \in \gamma, U(\alpha) \ll B U(\gamma)$; and

(3) for each $x \in X$, either $x \in \cup U(\gamma)$ or $\cup थ$ contains a base at $x$.

Proof. For each $\alpha \in \gamma$ and each $x \in \cup U(\alpha)$ let $b(x, \alpha) \in B$ such that there exists $u \in U(\alpha)$ with $\operatorname{St}(x, U(\alpha+1)) \subset b(x, \alpha) \subset \mathrm{Cl} b(x, \alpha)$ $\subset \neq u$, unless $u$ is an isolated point, in which case $b(x, \alpha)=\{x\}$. If $x \notin \cup U(\alpha)$, then let $b(x, \alpha)=X$.

For each $x \in X$, let $B(x)=\varepsilon b(x, \alpha)(\alpha \in \gamma)$ and let $U(\gamma)=\varepsilon \cap$ $B(x)(x \in X$ and $\cap B(x)$ is a non-empty open set). $U(\gamma)$ is a collection of open sets and since each $B(x)$ is perfectly decreasing, the elements of $U(\gamma)$ are also closed.

We now show that $U(\gamma)$ is pair-wise disjoint. First, suppose $x \neq y$ and there exists $\alpha \in \gamma$ such that $x \notin b(y, \alpha)$, then $x \notin \operatorname{St}(y, U(\alpha+1))$ and hence $y \notin \operatorname{St}(x, U(\alpha+1))$. It follows that $\operatorname{St}(x, U(\alpha+2)) \cap$ $\operatorname{St}(y, U(\alpha+2))=0$, since if there exists $z$ in this intersection, then $\operatorname{St}(z, U(\alpha+2))$ contains both $x$ and $y$, which is impossible since $\operatorname{St}(z, U(\alpha+2))$ is a subset of some element of $U(\alpha+1)$ and no element of $U(\alpha+1)$ contains both $x$ and $y$. Now this implies that $b(x, \alpha+2)$ $\cap b(y, \alpha+2)=0$ and thus we have $(\cap B(x)) \cap(\cap B(y))=0$. 
Second, let $x$ and $y$ be such that $\cap B(x) \neq \cap B(y)$ and let $z \in$ $\cap B(x)-\cap B(y)$. There exists $\alpha \in \gamma$ such that $z \notin b(y, \alpha)$ and hence $(\cap B(z)) \cap(\cap B(y))=0$, and since $z \in \cap B(x)$ we must have $x \in$ $\cap B(z)$, from which it follows that $(\cap B(x)) \cap(\cap B(y))=0$.

(2) holds, since for each $x \in \cup U(\gamma)$ and $\alpha \in \gamma$, we have $x \in v=$ $\mathrm{Cl} v \subset \mathrm{Cl} b(x, \alpha) \subset \neq u$, where $v \in U(\gamma)$ and $u \in U(\alpha)$.

(3) holds, since for each $x \in X$, if $x \notin \cup U(\gamma)$, then $B(x)$ is not open, implying that $\varepsilon b(x, \alpha)(\alpha \in \gamma)$ contains a base at $x$ and hence $\cup \mathcal{Q}$ contains a base at $x$.

THEOREM 1. If $X$ is a paracompact space with a perfectly monotone ortho-base $B$, then $X$ admits a proto-uniformizing family $\mathcal{U}=\mathcal{E} U(\alpha)(\alpha \in \gamma)$ such that:

(1) for each $\alpha \in \beta \in \gamma, U(\alpha) \ll B U(\beta)$; and

(2) for each $\alpha \in \gamma, U(\alpha)$ is a locally finite-in-itself collection of canonical open sets and if $\alpha$ is a limit ordinal, then $U(\alpha)$ is a pair-wise disjoint collection of closed-open sets.

Proof. We construct $\mathcal{Q}$ inductively, beginning with $U(0)=\{X\}$, using Lemmas 1 and 2 and the ordering $\ll B$. The fact that $\mathcal{Q}$ is a proto-uniformizing family having properties (1) and (2) follows directly from the conclusions of the lemmas.

This is a strengthening of a similar result of Gruenhage and Zenor [3, Lemma 2.3].

III. Trees and proto-metrizable spaces. A tree [5, p. 91] is an ordered set $(T,<)$ with the property that for each $t \in T, \mathcal{E} s(s<t)$ is isomorphic to an ordinal denoted ord $(t)$.

The length of the tree $T$ is len $(T)=\operatorname{lub}(\operatorname{ord}(t)+1)(t \in T) . T(\alpha)=$ $\varepsilon t(\operatorname{ord}(t)$ is isomorphic to $\alpha)$ is called the $\alpha$-th level of $T$ and if $T(x)$ is a tree, then $T(x, \alpha)$ is the $\alpha$-th level of $T(x)$.

A totally ordered subset of a tree is called a chain and a branch of a tree is a maximal chain, that is, a chain which is not a proper subset of some chain. For each $t \in T,[t]$ denotes the set of all branches containing $t$. The branch space of a tree $T$ is the set of all branches of $T$ with $\mathcal{E}[t]$ $(t \in T)$ as a basis and it follows from the definition of a tree that this is a rank-1 basis. A chain $b^{\prime}$ is said to be cofinal in the branch $b$ if for each $t \in b$ there exists $t^{\prime} \in b^{\prime}$ with $t \leq t^{\prime}$.

An element of a tree is said to be minimal with respect to some property if no element which precedes it has that property. When referring to a minimal element, it will be clear from the context which property is meant. 
Call a point $t$ of a tree inessential if either:

(1) $[t]$ has only one branch and $t$ is not minimal with respect to this property; or

(2) $t$ has exactly one immediate successor and $[t]$ has more than one branch.

A tree which contains no inessential point is said to be properly branching. If the inessential points are removed from a tree then the branch space of the resulting tree is homeomorphic to the branch space of the original tree, so for this reason we shall assume that a tree is properly branching unless stated otherwise.

If $\alpha$ is an ordinal or any well-ordered set, then the cofinality of $\alpha$, denoted $\operatorname{cf}(\alpha)$, is the least ordinal $\beta$ for which there exists a function $f$, called a cofinality function, from $\beta$ into $\alpha$ such that lub $f(\gamma)(\gamma \in \beta)=\alpha$. We shall always assume that a cofinality function is strictly order preserving. A cardinal $\kappa$ such that $\operatorname{cf}(\kappa)=\kappa$ is said to be a regular cardinal.

The character of a point $x$ of a topological space is the greatest cardinal $\chi(x)$ such that no local base for $x$ has cardinality less than $\chi(x)$. Clearly, if $b$ is a point of the branch space of a properly branching tree, then $\operatorname{cf}(b)=\chi(b)$, a regular cardinal.

An anti-chain in a tree $T$ is a subset $A$ which is pair-wise incomparable, that is, if $t, t^{\prime} \in A$, then neither $t<t^{\prime}$ nor $t^{\prime}<t$. An anti-chain is maximal if it is not a proper subset of some anti-chain.

If $A$ is an anti-chain of the tree $T$, then $\mathcal{E}[t](t \in A)$ is a collection of pair-wise disjoint closed-open sets in the branch space of $T$ and if this collection is a cover, then $A$ is called a covering anti-chain. A covering anti-chain is maximal, however the converse does not hold.

We extend the ordering $<$ on a tree $T$ to include its anti-chains: If $A$ and $A^{\prime}$ are anti-chains of $T$, then $A<A^{\prime}$ if and only if $A \neq A^{\prime}$ and for each $t^{\prime} \in A^{\prime}$ there exists $t \in A$ such that $t \leq t^{\prime}$. If $Q$ is a collection of anti-chains, then an anti-chain $C$ is said to be an upper bound for $Q$ if for each $A \in \mathbb{Q}, A \leq C$.

Let $T$ be a tree and let $S$ be a sub-tree of $T . S$ is a dense sub-tree if for each $t \in T$ there exists $s \in S$ with $t \leq s$ and $S$ is a cofinal sub-tree if for each branch $b \subset T, b \cap S$ is cofinal in $b$. A cofinal sub-tree is dense but the converse does not hold.

(When we refer to a level or branch of a sub-tree we mean with respect to the sub-tree and not to the tree in which it is embedded.)

(If $S$ is a subset of the branch space of a tree $T$, then $\cup S$ may contain branches which are not branches of $T$ even if $S$ is closed, thus when we say that a sub-tree is cofinal in $U S$ we mean only with respect to those branches which are branches of $T$.)

LEMMA 3. If $S$ is a cofinal sub-tree of the tree $T$, then the branch spaces of $S$ and $T$ are homeomorphic. 
Proof. For each branch $b \subset S$, there is a branch $\phi(b) \subset T$ with $b \subset \phi(b)$ and $\phi(b)$ is the only branch of $T$ which contains $b$, since $b$ must be cofinal in $\phi(b)$. Clearly $\phi$ is a homeomorphism.

Nyikos has shown that a space is non-archemedian if and only if it is an ultra-paracompact space with an ortho-base. (A space is ultra-paracompact if each open cover has a pair-wise disjoint open refinement.) It is also shown [12, Lemma 1.6] that a space is non-archemedian if and only if it has a base which is a tree under reverse set inclusion, thus we have:

A space is non-archemedian if and only if it is (densely) embeddable in the branch space of some tree. We will use this as our definition of non-archemedian space.

A subset $S$ of a topological space is defined [6, p. 1] as a $G_{\delta}(\alpha)$-set if $S$ is the intersection of a collection of open sets having cardinality no more than $\omega(\alpha)$. We will call $S$ a monotone $G_{\delta}(\alpha)$-set if $\alpha$ is the least ordinal such that $S$ is the intersection of a collection of open sets well-ordered by reverse set inclusion and the collection has cardinality $\omega(\alpha)$. Clearly, in the case of a monotone $G_{\delta}(\alpha)$-set, $\omega(\alpha)$ is a regular cardinal and each non-open $G_{\delta}$-set is a monotone $G_{\delta}(0)$-set.

A set $S$ is defined in [15] as finally $\omega(\alpha)$-compact if each open cover of $S$ contains a subcover of cardinality less than $\omega(\alpha)$. A finally $\omega$-compact set is just a compact set.

The following lemmas concern monotone $G_{\delta}(\alpha)$-sets and compact $G_{\delta}$-sets in non-archemedian spaces.

Lemma 4A. Suppose $S$ is a nowhere dense (i.e. In $\mathrm{Cl} S=0$ ) subset of the branch space of a tree. If $S$ is a monotone $G_{\delta}(\alpha)$-set, then $\cup S$ contains a cofinal sub-tree whose branches have the same length and cofinality $\omega(\alpha)$ and conversely if $S$ is closed.

Proof. Suppose $S$ is a monotone $G_{\delta}(\alpha)$-set and let $\mathscr{E} G(\beta)(\beta \in \omega(\alpha))$ be the decreasing sequence of open sets whose intersection is $S$. For each $\beta \in \omega(\alpha)$, let $A(\beta)$ be the anti-chain of $\cup S$ consisting of the minimal elements $t \in \cup S$ such that $[t] \subset G(\beta)$, then we have $A(\beta) \leq A(\gamma)$ for $\beta \in \gamma$ so $\mathcal{E} A(\beta)(\beta \in \omega(\alpha))$ is a well-ordered increasing sequence of covering anti-chains of $\cup S$. Since $S$ is nowhere dense, no element of $\cup S$ is an upper bound for this collection of anti-chains, hence its union is a cofinal sub-tree of $\cup S$ and its branches must have length $\omega(\alpha)$.

If $S$ is closed and $\cup S$ contains a cofinal sub-tree $R$ whose branches have the same length $\gamma$ and cofinality $\omega(\alpha)$, then let $f$ be a cofinality function from $\omega(\alpha)$ into $\gamma$ and for each $\beta \in \omega(\alpha)$, let $G(\beta)=\cup[t]$ $(t \in R(f(\beta)))$ so that $S=\cap G(\beta)(\beta \in \omega(\alpha))$. 
The following is a well-known lemma which is found in [5, p. 91]:

Konig's Lemma. If $T$ is a tree of length $\omega$ and if each level of $T$ is finite, then $T$ has a branch of length $\omega$.

LEMMA 4B. Suppose $S$ is a nowhere dense subset of the branch space of a tree, then $S$ is a compact $G_{\delta}$-set if and only if $S$ is closed and $\cup S$ contains $a$ cofinal sub-tree such that each branch has length $\omega$ and each level is finite.

Proof. Suppose $S$ is a compact $G_{\delta}$-set, then $S$ is a monotone $G_{\delta}$-set and by Lemma $4 \mathrm{~A}$ there exists a cofinal sub-tree $R$ of $\cup S$ such that each of its branches has the same length $\gamma$ and cofinality $\omega$. Let $f$ be a cofinality function from $\omega$ into $\gamma$ and let $R^{\prime}=\cup R(f(\beta))(\beta \in \omega)$, then $R^{\prime}$ is also a cofinal sub-tree of $\cup S$, its branches have length $\omega$ and since $S$ is compact, its levels must be finite.

Conversely, suppose $S$ is closed, each branch of $\cup S$ has length $\omega$ and that each level is finite, then by Lemma $4 \mathrm{~A}, S$ is a $G_{\delta}$-set and using Konig's Lemma it is easily seen that each open cover of $S$ is refined by the cover $\mathcal{E}[t](t \in L)$ for some level $L$ of $\cup S$, hence $S$ is compact.

LEMMA 4C. If the branch space of a properly branching tree is finally $\omega(\alpha)$-compact, then each chain in the tree which is not cofinal in a branch must have cofinality less than $\omega(\alpha)$.

Proof. If the chain $c$ is not cofinal in a branch then there is an element of the tree which is the least upper bound of $c$. If the cofinality of $c$ is not less than $\omega(\alpha)$, then there exists an anti-chain in the tree having $\omega(\alpha)$ elements which can be extended to a covering anti-chain since $c$ has an upper bound. This is clearly impossible if the branch space is finally $\omega(\alpha)$-compact.

Suppose $X$ is a space and $T$ is a collection of closed subsets of $X$ which is a tree when ordered by reverse set inclusion. A branch $b$ of $T$ is said to converge to $x \in X$ if for each open set $u$ containing $x$ there exists $t \in b$ such that $t \subset u$. Note that if $X$ is a Hausdorff space, then no branch converges to two distinct points of $X$. If for each $x \in X$ there exists a branch of $T$ which converges to $x$ and if each branch of $T$ either converges to a point of $X$ or has empty intersection, then $T$ is said to be cofinal in $X$. If for each $x \in X$ and each open $u$ containing $x$ there exists $t \in T$ such that $t \subset u$, then $T$ is said to be dense in $X$.

A partition of a space $X$ is a collection of canonical closed sets which covers $X$ and such that the interiors of two distinct elements do not intersect. 
THEOREM 2. If $X$ is a paracompact space with a perfectly monotone ortho-base, then $X$ admits a cofinal tree of canonical closed subsets such that each level is locally finite-in-itself and each limit level is a pair-wise disjoint collection of closed-open sets.

Proof. Let $B$ be a perfectly monotone ortho-base for $X$ and let $\mathcal{Q}=\mathcal{E} U(\alpha)(\alpha \in \gamma)$ be the proto-uniformizing family with ordering $\ll B$ constructed in Theorem 1. For each $\alpha \in \gamma$, let $\alpha^{\prime}$ denote the limit ordinal and $n(\alpha)$ the finite ordinal such that $\alpha=\alpha^{\prime}+n(\alpha)$ and let $U^{\prime}(\alpha)=$ $\cup U\left(\alpha^{\prime}+i\right)(i \in n(\alpha)+1)$.

For each $x \in \cup U^{\prime}(\alpha)$, let

$$
p(x, \alpha)=\mathrm{Cl}\left[\bigcap u \in U^{\prime}(\alpha)(x \in u)-\mathrm{Cl} \bigcup u \in U^{\prime}(\alpha)(x \notin u)\right]
$$

and let $P(\alpha)=\mathcal{E} p(x, \alpha)\left(p(x, \alpha) \neq 0\right.$ and $\left.x \in \cup U^{\prime}(\alpha)\right)$. For each $\alpha \in \gamma$, $P(\alpha)$ is a partition of $\cup U\left(\alpha^{\prime}\right)$ and since $U^{\prime}(\alpha)$ is locally finite-in-itself, then so is $P(\alpha)$.

We may not obtain a tree directly from this sequence of partitions, however, it follows from the construction of $\mathscr{Q}$ that the union of limit elements of $Q$ is a tree and it is evident from the construction above that for each $\alpha, \cup P(\alpha+i)(i \in \omega)$ is a tree.

For each limit ordinal $\alpha$ and each $u \in P(\alpha+\omega)$, let $c(u)$ be a branch of $\cup P(\alpha+i)(i \in \omega)$ such that $\cap c(u) \subset u$ and then for each $\alpha \in \gamma$ and $p \in P(\alpha)$ let

$$
t(p)=\mathrm{C} \operatorname{In}[(p-\bigcup P(\alpha+\omega)) \cup \bigcup u \in P(\alpha+\omega)(p \in c(u))] .
$$

We now construct a tree $T$ of canonical closed subsets of $X$ by requiring that for each $\alpha \in \gamma$ the $\alpha$-th level $T(\alpha)=\delta t(p)(p \in P(\alpha)$ and $t(p) \neq 0)$. This tree may not be properly branching but it is more convenient to use when showing cofinality. (Use $T=\varepsilon t(p)(p \in \cup P(\alpha)$ $(\alpha \in \gamma)$ and $t(p) \neq 0$ ) for a properly branching tree.)

We must now show that $T$ is cofinal in $X$. For each $x \in X$ and $\alpha \in \gamma$, choose $t(x, \alpha) \in T(\alpha)$ such that $x \in t(x, \alpha) \subset \cap t(x, \beta)(\beta \in \alpha)$ if it exists, otherwise let $t(x, \alpha)=t(x, 0)=X$. $\delta t(x, \alpha)(\alpha \in \gamma)$ is a branch of $T$ (but perhaps not of length $\gamma$ ) and we must show that this branch converges to $x$.

Let $v$ be an open set containing $x$ and recall the construction used in Lemma 2 where it is shown that the limit elements of $\mathcal{Q}$ are pair-wise disjoint. There exists $\alpha \in \gamma$ such that $\operatorname{St}(x, U(\alpha)) \subset v$. Let $p \in P(\alpha+3)$ such that $t(p) \in T(\alpha+3), x \in t(p)$ and suppose there exists $u \in$ $U(\alpha+\omega)$ such that $p \in c(u)$, hence $u \subset t(p)$ and $p \cap u \neq 0$. Let $z \in p$ $\cap u$ and assume that $t(p)$ is not a subset of $\operatorname{St}(x, U(\alpha))$, that is, there exists $y \in u$ with $y \notin \operatorname{St}(x, U(\alpha))$. Thus we now have points $z$ and $y$ with 
$y \neq z$ and $y \notin b(z, \alpha+1)$. It follows from the construction in Lemma 2 that $y \notin u$, contradicting the assumption, hence $t(p) \subset \operatorname{St}(x, U(\alpha)) \subset v$ and the branch $\varepsilon t(x, \alpha)(\alpha \in \gamma)$ converges to $x$.

Suppose the branch $b$ of $T$ does not converge to a point of $X$. Let $\beta$ be the least ordinal such that $b \cap T(\beta)=0$ and for each $\alpha \in \beta$ let $x(\alpha) \in b$ $\cap T(\alpha)$. Now using the notation of Lemma 2 , let $B^{\prime}=\delta b(x(\alpha), \alpha)$ $(\alpha \in \beta) \subset B$. Either $\cap B^{\prime}=0$ or $\cap B^{\prime}$ is an element $u$ of a limit level $T(\alpha+\omega)$ of $T$ in which case there is a branch $b^{\prime}$ of $\cup P\left(\alpha^{\prime}+i\right)(i \in \omega)$ such that some final segment of $b$ is $\varepsilon t(p)\left(p \in b^{\prime}\right)$ but $c(u) \neq b^{\prime}$. In either case $\cap b=0$.

A continuous function or map is said to be closed if the image of each closed set is closed. We denote the image of a set $H$ under $f$ by $f H$, that is, $f H=\mathcal{E} f(x)(x \in H)$ and the inverse of $f$ is denoted by $\hat{f}$ so that $\hat{f} H=\mathcal{E} x(f(x) \in H)$.

A continuous function from $Y$ onto $X$ is irreducible if no proper closed subset of $Y$ maps onto $X$; equivalently, each open subset of $Y$ contains the pre-image of some point of $X$.

Suppose $Y$ is a subspace of the branch space of a tree $T$ (a non-archemedian space) and $\phi$ is a closed map from $Y$ onto $X$. We shall use the term "generates" to describe each of the following procedures:

(1) If $A$ is an anti-chain in $T$, then $A$ is said to generate the open sets $\cup[t](t \in A) \subset Y$ and In $\cup \phi[t](t \in A) \subset X$.

(2) If $u$ is open in $X$, then the anti-chain $A$ consisting only of all minimal $t \in T$ such that $[t] \subset \hat{\phi} u$ is said to be generated by $u$. $u$ is also said to generate $\cup[t](t \in A) \subset Y$ if $u$ generates $A$.

THEOREM 3. If the regular Hausdorff space $X$ admits a cofinal tree $T$ of canonical closed sets such that each level of $T$ is locally finite-in-itself and limit levels are pair-wise disjoint collections of closed-open sets, then $X$ is the irreducible closed continuous image of a (dense) subspace of the branch space of $T$ (a non-archemedian space) such that each point pre-image either has only one branch or is a compact $G_{\delta}$-set having branches of equal length.

Proof. Let $Y$ be the sub-branch space of $T$ consisting only of all branches which converge to some point of $X$ and let $\phi$ be the function from $Y$ to $X$ such that for each $y \in Y, \phi(y)$ is the point of $X$ to which $y$ converges. Since $T$ is cofinal in $X, \phi$ is onto.

(A) For each $x \in X$, the branches of $\hat{\phi(x)}$ have the same length.

Suppose $y, y^{\prime} \in \hat{\phi(x)}$ and assume that $\operatorname{len}(y) \in \operatorname{len}\left(y^{\prime}\right)$. Let $\alpha$ be the least ordinal such that $y^{\prime} \cap T(\alpha) \neq 0$ and $y \cap T(\alpha)=0$ and let $\beta$ be the least ordinal such that $T(\beta) \cap y \neq T(\beta) \cap y^{\prime} . \alpha$ is a limit ordinal, since if not, then $y$ and $y^{\prime}$ both have an $(\alpha-1)$-st element which are canonical 
closed sets with disjoint interiors and both contain $x$; but the $(\alpha-1)$-st element of $y$ is $\{x\}$, since it is the last element of $y$, a contradiction.

Notice that since the collection $T$ is a tree under ordering by reverse set inclusion and since $T$ is cofinal in $X$, each level of $T$ must be a partition of its union. $T(\beta) \cap y$ and $T(\beta) \cap y^{\prime}$ are closed sets each containing $x$ as a boundary point. $T(\alpha) \cap y^{\prime}$ is a closed-open set containing $x$ and is a subset of $T(\beta) \cap y^{\prime}$, hence $\operatorname{In}\left(T(\beta) \cap y^{\prime}\right) \cap \operatorname{In}(T(\beta) \cap y)$ $\neq 0$, which is impossible as noted above and contradicts our assumption.

(B) $\phi$ is continuous.

Suppose $g$ is an open subset of $X$. Since $X$ is regular, for each branch $b \in \hat{\phi g}=U \hat{\phi}(x)(x \in g)$, there exists $t(b) \in b$ which is the minimal element of $b$ that is a subset of $g . G=\cup[t(b)](b \in \hat{\phi} g)$ is an open set in the branch space of $T$. For each $x \in g, \hat{\phi}(x) \subset G \cap Y$ and conversely each branch $b \in G \cap Y$ must converge to some $x \in g$, hence $\hat{\phi} g=G \cap Y$ is an open set in $Y$. We have shown that $\hat{\phi}$ preserves open sets and hence $\phi$ is continuous.

(C) $\phi$ is irreducible.

We must show that each open set in $Y$ contains $\hat{\phi(x)}$ for some $x \in X$. Suppose $t \in T$, then $[t]$ is a basic open set in the branch space and $t$ is a

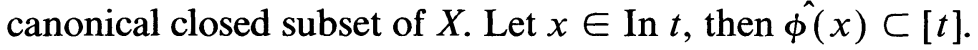

(D) Each point pre-image either has only one branch or is a compact $G_{\delta}$-set .

Suppose $x \in X$ and $\hat{\phi(x)}$ has more than one branch. We have already shown that the branches of $\hat{\phi}(x)$ have the same length. The ordinal len $\cup \hat{\phi(} x)$ cannot contain a cofinal sequence of limit ordinals, since the limit levels of $T$ are pair-wise disjoint collections of closed-open sets, hence each branch of $\phi(x)$ has a final segment isomorphic to $\omega$.

Let $T(x)$ be the union of all final $\omega$-segments of branches of $\cup \hat{\phi(x)}$, then $T(x)$ is a tree of length $\omega$ with finite levels and is cofinal in $\cup \hat{\phi(x)}$.

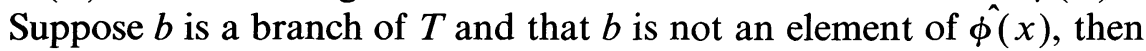
either $b$ converges to a point other than $x$ or $\cap b=0$, and in either case there exists an element $t \in b$ which does not contain $x$, that is, $[t] \cap \hat{\phi(}(x)$ $=0$, hence $\hat{\phi(x)}$ is closed in the branch space of $T$. Further, since $\phi$ is irreducible, $\hat{\phi(x)}$ is nowhere dense and now, using Lemma $4 \mathrm{~B}, \hat{\phi(x)}$ is a compact $G_{\delta}$-set.

(E) $\phi$ is a closed map.

Suppose $H$ is a closed subset of $Y$ and $x \in X$ is not an element of $\phi H$. $\hat{\phi(x)}$ is compact and does not intersect the closure of $H$ in the branch space of $T$, hence there exists a finite level $L$ of $\cup \hat{\phi(}(x)$, a sub-level of $T$, such that for each $t \in L,[t] \cap \mathrm{Cl} H=0$.

Recall that the tree $T$ is a collection of canonical closed subsets of $X$, thus In $\cup t(t \in L)$ is an open set containing $x$ which does not intersect $\phi H$, hence $\phi H$ is closed and $\phi$ is a closed map. 
The following definitions are necessary for the next theorem. Suppose $\phi$ is a closed map from a sub-branch space of the tree $T$ onto the space $X$. For each $x \in X$, the sub-tree $\cup \hat{\phi(x)}$ is denoted simply by $T(x)$ and it is understood that a covering anti-chain of $T(x)$ must be a subset of $T(x)$. For each anti-chain $A$ of $T$, let $[A]$ (or $[A, \phi]$ if necessary for clarity) denote the set consisting only of all $x \in X$ such that $A$ is a covering anti-chain of $T(x)$. Note that this notation is consistent with the notation $[t]$ introduced earlier.

Two branches of a tree are said to split at $t$ if $[t]$ contains both branches but no element following $t$ is an element of both branches. For each $x \in X$, those elements of $T(x)$ at which two branches of $T(x)$ split are called the splitting elements of $T(x)$.

THEOREM 4. If $X$ is the (irreducible) closed continuous image of a sub-branch space of a tree ( $a$ non-archemedian space) such that for each point of $X$ there is an ordinal $\alpha$ such that the pre-image of this point is a finally $\omega(\alpha)$-compact monotone $G_{\delta}(\alpha)$-set, then $X$ is a paracompact space with a perfectly monotone ortho-base.

Proof. That $X$ is paracompact follows from the result of Michael [8] that the closed continuous image of a paracompact space is paracompact.

Let $T$ be a tree whose branch space contains the pre-image of $X$ where $\phi$ is the map with the specified properties including the assumption that it is irreducible. It will be a consequence of Lemma 5 and the hereditary nature of proto-metrizability discussed below that irreducibility is not necessary here.

We will now construct, for each $x \in X$, a sequence $S(x)$ of covering anti-chains of $T(x)$. Note that the $\alpha$-th element of $S(x)$ will be denoted by $S(x, \alpha)$ and this may not be the $\alpha$-th level of the tree $\cup S(x)$. The construction is as follows.

(1) Since $\phi$ is irreducible, it follows from Lemma 4A that $T(x)$ admits a cofinal sub-tree $S^{\prime}(x)$ whose branches have length $\chi(x)$.

(2) Let $S^{\prime \prime}(x)$ be the set of splitting elements of $T(x) \cdot S^{\prime \prime}(x)$ may not be cofinal in $T(x)$ and may be empty. It is a consequence of Lemma 4C that no branch of $S^{\prime \prime}(x)$ has length greater than $\chi(x)$.

(3) Now construct, inductively, a cofinal sub-tree $S^{\prime \prime \prime}(x)$ of $T(x)$ by extending the (perhaps empty) levels of $S^{\prime \prime}(x)$ to covering anti-chains of $T(x)$ so that for each $\alpha \in \chi(x)$ : (a) $S^{\prime \prime}(x, \alpha) \subset S^{\prime \prime \prime}(x, \alpha)$; (b) each element of $S^{\prime \prime \prime}(x, \alpha)-S^{\prime \prime}(x, \alpha)$ follows $S^{\prime}(x, \alpha)$; and (c) $\alpha \in \beta$ implies that $S^{\prime \prime \prime}(x, \alpha)<S^{\prime \prime \prime}(x, \beta)$ and $S^{\prime \prime \prime}(x, \alpha) \cap S^{\prime \prime \prime}(x, \beta)=0$.

(4) The anti-chain sequence, $S(x)$, is now constructed so that for each $\alpha \in \chi(x)$ either

(a) if $\left[S^{\prime \prime \prime}(x, \alpha)\right]=\{x\}$, then $S(x, \alpha)=S^{\prime \prime \prime}(x, \alpha)$; or 
(b) if $\left[S^{\prime \prime \prime}(x, \alpha)\right]$ contains at least two points, then choose $x^{\prime} \in$ $\left[S^{\prime \prime \prime}(x, \alpha)\right]-\{x\}$. Let $S(x, \alpha)$ contain $t$ if and only if $t \in S^{\prime \prime}(x, \alpha)$ or there exists a branch $b$ of $T(x)$ such that $b$ contains no element of $S^{\prime \prime}(x, \alpha)$ and $t$ is the least element of $b$ which is not an element of $T\left(x^{\prime}\right)$.

We now construct a base for $X$. For each $x \in X$ and $\alpha \in \chi(x)$, let $b(x, \alpha)=\operatorname{In} \cup \phi[t](t \in S(x, \alpha))$ and let $B=\mathcal{E} b(x, \alpha)(x \in X$ and $\alpha \in$ $\chi(x)$ ). Each open set containing $x$ contains an open set generated by a covering anti-chain $A$ of $T(x)$. Since $\hat{\phi(x)}$ is finally $\chi(x)$-compact, $A$ has less than $\chi(x)$ elements, so there exist anti-chains $S^{\prime}(x, \alpha)$ and (perhaps) $S^{\prime \prime}(x, \alpha)$ with $A<S^{\prime}(x, \alpha)$ and $A<S^{\prime \prime}(x, \alpha)$, and it follows from (3) and (4) in the construction of $S(x)$ that $A<S(x, \alpha)$, hence $B$ is a base for $X$.

We must now show that $B$ is a perfectly monotone ortho-base for $X$. Let $B^{\prime}$ be a perfectly decreasing subset of $B$ and suppose there exists $x \in \cap B^{\prime}-$ In $\cap B^{\prime}$. It remains only to show that $B^{\prime}$ contains a base at $x$.

Each element of $B^{\prime}$ is of the form $b(y, \beta)$ which is generated by an anti-chain of the form $S(y, \beta)$, so we let $Y=\mathcal{E} y\left(b(y, \beta) \in B^{\prime}\right)$ and let $Q=\mathcal{E} S(y, \beta)\left(b(y, \beta) \in B^{\prime}\right)$. Since $B^{\prime}$ is perfectly decreasing and $\phi$ is irreducible, $\mathbb{Q}$ is a sequence of anti-chains well-ordered by $<$ and we let $Y$ have the corresponding well-ordering.

Since $x \notin$ In $\cap B^{\prime}$, the anti-chain sequence $\mathbb{Q}$ must be cofinal in some branch $b^{\prime}$ of $T(x)$, that is, $\mathscr{E} b^{\prime} \cap A(A \in \mathbb{Q})$ is cofinal in $b^{\prime}$. This holds for each boundary point of $\cap B^{\prime}$ and with the construction of $B$ implies

(A) $\cap B^{\prime}$ can have at most one boundary point, which is $x$ and $x$ is a limit point of each cofinal sub-sequence of $Y$.

Let $U=\delta t$ ( $t$ is minimal with respect to the property that for each $A \in \mathbb{Q}, A<\{t\})$. Clearly, if $U$ is not empty, then $U$ is an anti-chain and $\cup \phi[t](t \in U)=\cap B^{\prime}$, thus $U \neq 0$ implies that $B^{\prime}$ does not contain a base at $x$ and since $\phi$ is a closed map the converse also holds. $U \neq 0$ also implies that some final segment of $Y$ must consist only of points distinct from $x$.

Under the assumption that $U$ is not empty, we prove the following statements.

(B) For each $u \in U$, there exists a final segment $Y^{\prime}$ of $Y$ such that for each $y \in Y^{\prime},[u]$ contains a branch of $\hat{\phi}(y)$.

Let $u \in U$. If there exists $A \in \mathbb{Q}$ with $u \in A$, we are finished. Let $S$ be an anti-chain of $T(x)$ no element of which precedes $u$. Assume that $u$ does not have the required property, then there exists a cofinal subsequence $Y^{\prime \prime}$ of $Y$ such that for each $y \in Y^{\prime \prime},[u]$ contains no branch of $\hat{\phi}(y)$ and such that $Y^{\prime \prime}$ is a subset of the open set generated by $S$. 
It follows from the definition of $U$ that for each $t<u$ there exists $A \in \mathbb{Q}$ and associated $y \in Y^{\prime \prime}$ such that some branch of $\hat{\phi}(y)$ contains $t$ but none contain $u$. Since $S$ must cover $\hat{\phi}(y)$, there exists a branch of $T(x)$ having this property also. Since $A$ is a $\chi(x)$-sequence, this implies that $u$ is preceded by a $\chi(x)$-sequence of splitting elements of $T(x)$ which contradicts Lemma $4 \mathrm{C}$.

(C) $U$ is a maximal (but non-covering) anti-chain of $T(x)$.

First we show that $U \subset T(x)$. If there exists $u \in U-T(x)$, then $\phi[u]$ is a closed set not containing $x$ and it follows from (B) that there is a final segment of $Y$ which is a subset of $\phi[u]$ but not having $x$ as a limit point, contradicting (A).

If $U$ is not maximal, then there exists $t \in T(x)$ such that $U \cup\{t\}$ is an anti-chain. Since the anti-chains in $\mathbb{Q}$ must cover $T(x)$, either $t$ follows some element of $U$ (which is not the case here) or $Q$ is cofinal with each branch of $T(x)$ which contains $t$, thus some final segment of $Y$ consists of boundary points of $\cap B^{\prime}$. Since $x$ is the only boundary point of $\cap B^{\prime}$, the construction of $B$ implies that $B^{\prime}$ contains a base at $x$, contradicting the assumption that $U$ is not empty. $U$ is non-covering due to the existence of the branch $b^{\prime}$.

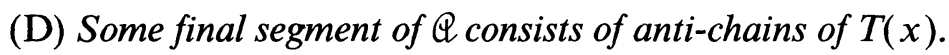

Assume to the contrary that there exists a cofinal subsequence $\mathbb{Q}^{\prime}$ of $\mathbb{Q}$ having the property that for each $A \in \mathbb{Q}^{\prime}, A$ is not a subset of $T(x)$, then for each $A \in Q^{\prime}$, there exists $t \in A$ such that (In $\left.\cap B^{\prime}\right) \cap \phi[t]=0$ since $U \subset T(x)$. It follows from (C) that the cofinal subsequence $Y^{\prime}$ of $Y$ associated with $\mathbb{Q}^{\prime}$ must have a final segment of boundary points of $\cap B^{\prime}$, again contradicting the assumption that $U$ is not empty.

(E) For each $\beta \in \chi(x)$, there is a $\beta$-sequence $V$ of splitting elements of $T(x)$ and a final segment $Y^{\prime}$ of $Y$ such that for each $y \in Y^{\prime}, V$ is a sequence of splitting elements of $T(y)$.

It follows from the construction of $b^{\prime}$ that $S^{\prime \prime}(x)$ is cofinal in $b^{\prime}$, so let $V(\beta)=\mathcal{E}\left(b^{\prime} \cap S^{\prime \prime}(x, \alpha)\right)(\alpha \in \beta)$. Note that $V(0)=0$, so statement (E) holds for $\beta=0, V(0)$ and $Y^{\prime}=Y$.

If $\beta$ is a limit ordinal and for each $\alpha \in \beta$, statement (E) holds for $V(\alpha)$ and final segment $Y^{\prime}(\alpha)$ of $Y$ where the anti-chains associated with $Y^{\prime}(\alpha)$ satisfy (D), then the statement holds for $V(\beta)=\bigcup V(\alpha)(\alpha \in \beta)$ and final segment $Y^{\prime}(\beta)=\bigcap Y^{\prime}(\alpha)(\alpha \in \beta)$.

Suppose the statement holds for $\beta, V(\beta)$ and $Y^{\prime}(\beta)$ where the anti-chains associated with $Y^{\prime}(\beta)$ satisfy (D). Let $s$ be the least splitting element of $T(x)$ in $b^{\prime}$ not contained in $V(\beta)$, then $V(\beta+1)=V(\beta) \cup$ $\{s\}$. There exists a branch $b$ of $T(x)$ and $u \in U$ such that $b$ and $b^{\prime}$ split at 
$s$ and $u \in b$. Let $Y^{\prime \prime}(\beta+1)$ be a final segment of $Y$ satisfying statement (B) for $u$ and such that for each $y \in Y^{\prime \prime}(\beta+1)$, s precedes an element of the associated anti-chain. The statement now holds for $V(\beta+1)$ and $Y^{\prime}(\beta+1)=Y^{\prime}(\beta) \cap Y^{\prime \prime}(\beta+1)$.

(F) For each $u \in U$, there exists $r<u$ and a final segment $Y^{\prime}$ of $Y$ such that for each $y \in Y^{\prime}$ there is exactly one branch of $\hat{\phi(y)}$ which contains $r$.

Suppose $u \in U$, then $u \in T(x)$. Let $\alpha$ be the length of the sequence of splitting elements of $T(x)$ which precede $u$ and let $\beta$ be an ordinal with $\alpha \in \beta$.

Let $Q^{\prime}$ be a final segment of $\mathcal{Q}$ and $Y^{\prime}$ the associated final segment of $Y$ such that (B) holds for $u,(\mathrm{D})$ holds for $Q^{\prime}$ and (E) holds for $Y^{\prime}$ and $\beta$.

It follows from the construction of $U$ and (3a) in the construction of $B$ that if $y \in Y^{\prime}$, then no branch of $\hat{\phi(y)}$ which contains $u$ can contain a splitting element of $T(y)$ which follows $u$, since $T(y)$ contains a $\beta$-sequence of splitting elements preceding the anti-chain associated with $y$. In fact, (3c) implies that if $r$ is the least upper bound of the $\alpha$-sequence, then $u \neq r$, so $r<u$ and $r$ has the desired properties.

We are now ready to use these statements to contradict $U \neq 0$. Let $u \in U$ and let $R$ be a covering anti-chain of $T(x)$ such that $u$ precedes some element of $R$. Let $\mathbb{Q}^{\prime}$ be a final segment of $\mathcal{Q}, Y^{\prime}$ the associated final segment of $Y$ and $r<u$ such that statements (B), (D) and (F) hold and $r$ precedes an element of each anti-chain in $\mathbb{Q}^{\prime}$.

As a consequence of statement (D), for each $A \in Q^{\prime},[A]$ contains both $x$ and the associated $y \in Y^{\prime}$. Since we may assume $y \neq x$, (4a) in the construction of $B$ cannot apply to $A$, hence (4b) must apply, so for each $A \in \mathbb{Q}^{\prime}$, there exists $Z(A) \in X$ such that some branch of $\hat{\phi(Z(A)) ~ c o n-~}$ tains $r$ but not $u$. Statement (F) implies that this branch of $Z(A)$ contains no element of $R$, hence, since $\phi$ is closed, the open set geneated by $R$ contains $x$ but no point of $\mathcal{E} Z(A)\left(A \in \mathbb{Q}^{\prime}\right)$.

But it is also true, since $Q$ is cofinal in $b^{\prime}$, that for each covering anti-chain $R$ of $T(x)$ (which generates an open set containing $x$ ), there exists a final segment $\mathbb{Q}^{\prime \prime}$ of $\mathbb{Q}^{\prime}$ such that for each $A \in \mathbb{Q}^{\prime \prime}$ there is a branch of $\hat{\phi(Z(A))}$ which contains $R \cap b^{\prime}$, hence each open set containing $x$ must contain $E Z(A)\left(A \in \mathbb{Q}^{\prime \prime}\right)$ since $\phi$ is closed. Under the assumption that $U \neq 0$ we have now obtained a contradiction, hence $B^{\prime}$ contains a base at $x$ and $B$ is a perfectly monotone ortho-base.

THEOREM 5. If $X$ is the (irreducible) closed continuous image of a sub-branch space of tree ( $a$ non-archemedian space) such that each point pre-image either has only one branch or is a compact $G_{\delta}$-set, then $X$ is proto-metrizable. 
Proof. We may assume that the map is irreducible since if not, then consider an ultra-filter $F$ of proper closed sub-sets of the space on which the map is defined and whose image is $X$. Since point pre-images are compact, $\cap F$ must intersect each point pre-image and the restriction of the map to $\cap F$ is irreducible.

Using the previous theorem, $X$ is a paracompact space with a perfectly monotone ortho-base, and now using Theorems 1, 2 and 3 we can obtain a tree $T$ and an irreducible closed map $\phi$ from a sub-branch space of $T$ onto $X$ such that each point pre-image either has only one branch or is a compact $G_{\delta}$-set having branches of equal length.

Here we generate basic open sets from sub-levels of $T$, thus for each $x \in X$, let $b(x, \alpha)=\operatorname{In} \cup \phi[t](t \in T(x, \alpha))$ where $T(x, \alpha)=T(x) \cap$ $T(\alpha)$, and let $B=\mathcal{E} b(x, \alpha)(x \in X$ and $\alpha \in$ len $T(x))$.

If $B^{\prime} \subset B$ and $x \in \cap B^{\prime}-$ In $\cap B^{\prime}$, then it follows from statement (A) in the proof of Theorem 4, the fact that $\phi$ is closed and the generation of basic open sets from sublevels, that $B^{\prime}$ contains a base at $x$ without requiring that $B^{\prime}$ be totally ordered. Hence $B$ is an ortho-base and $X$ is proto-metrizable.

Using the fact that a non-archemedian space is the sub-branch space of a tree, it is easy to see that the property of being non-archemedian is hereditary. Using this with the characterization of proto-metrizable spaces obtained in Theorem 5, it is easily seen, by restricting the domain of the map, that proto-metrizability is hereditary and thus proto-metrizable spaces are hereditarily paracompact. This is a result of Gruenhage and Zenor [3, Lemma 2.2] and is also stated by Nyikos [12].

The hereditary nature of proto-metrizability and the following lemma allow us to remove the condition of irreducibility from Theorem 4. It does not, however, imply that the map used in Theorem 4 may be made irreducible by restricting its domain, as was done in Theorem 5 .

LEMMA 5. Suppose $\phi$ is a closed map from the sub-branch space $Y$ of the tree $T$ onto $X$, then there exists an irreducible closed map $\phi^{\prime}$ from the sub-branch space $Y^{\prime}$ of a tree $T^{\prime}$ onto $X^{\prime}$ and $X^{\prime}$ contains a closed nowhere dense copy of $X$. Further, if the point pre-images of $\phi$ are finally $\omega(\alpha)$-compact monotone $G_{\delta}(\alpha)$-sets, then so are the point pre-images of $\phi^{\prime}$.

Proof. Since an isolated branch of a tree may be extended without changing the topology of the branch space, we shall assume that each branch of $T$ is isomorphic to a limit ordinal. Of course $T$ may no longer be a properly branching tree. We shall also assume that point pre-images under $\phi$ are nowhere dense. 
Let $<$ be the ordering on $T$, let $T^{\prime}=T \times\{0,1\}$ and extend the order $<$ to $T^{\prime}$ so that: (a) $(t, 0)<\left(t^{\prime}, 0\right)$ if and only if $t<t^{\prime}$; (b) $(t, 0)<(t, 1)$ for each $t \in T$; and (c) for each $t, t^{\prime} \in T$ neither $(t, 1)<\left(t^{\prime}, 1\right)$ nor $\left(t^{\prime}, 1\right)<(t, 1)$.

Notice that the branch space of $T^{\prime}$ contains a copy of $Y$ consisting of the branches of $T \times\{0\}$ identified with the branches of $Y$. $Y^{\prime}$ is the sub-branch space of $T^{\prime}$ obtained by taking the union of $Y$ and the set of all branches with end-point in $T \times\{1\}$. $Y$ is now a closed nowhere dense subset of $Y^{\prime}$.

$\phi^{\prime}$ is the map defined on $Y^{\prime}$ so that its restriction to $Y$ is $\phi$ and its restriction to $Y^{\prime}-Y$ is the identity and $X^{\prime}=X \cup\left(Y^{\prime}-Y\right)$ where a set is open in $X^{\prime}$ if and only if it is the image under $\phi^{\prime}$ of an open set in $Y^{\prime}$ whose intersection with $X$ is either open in $X$ or is empty. It follows that $X$ is a closed nowhere dense subset of $X^{\prime}$ and that $\phi^{\prime}$ is a closed irreducible map.

Further, under the assumption that point pre-images under $\phi$ are nowhere dense, if point pre-images of $\phi$ are finally $\omega(\alpha)$-compact monotone $G_{\delta}(\alpha)$-sets, then $\phi^{\prime}$ retains this property. This result remains valid even when point pre-images are not nowhere dense since, if necessary, we can restrict the domain of $\phi$ so that it is defined only at those points which are not in the interior of some point pre-image or in case of an isolated point choose a point from its pre-image. Since the images of $\phi$ and of this restriction are homeomorphic, we can replace $\phi$ with this restriction of $\phi$ in the above proof.

We summarize the previous results with:

THEOREM 6. The following are equivalent for a regular Hausdorff space $X$ :

(a) $X$ is proto-metrizable;

(b) $X$ is a paracompact space with an ortho-base;

(c) $X$ is a paracompact space with a (perfectly) monotone ortho-base;

(d) $X$ admits a proto-uniformizing family well-ordered by refinement ( such that each element is a locally finite-in-itself collection of canonical open sets and each limit element is a pair-wise disjoint collection of closed open sets);

(e) $X$ admits a cofinal tree of canonical closed subsets such that each level is locally finite-in-itself and limit levels are pair-wise disjoint collections of closed-open sets;

(f) $X$ is the (irreducible) closed continuous image of a non-archemedian space such that for each point of $X$ there is an ordinal $\alpha$ such that the pre-image of the point is a finally $\omega(\alpha)$-compact monotone $G_{\delta}(\alpha)$-set; 
(g) $X$ is the (irreducible) closed continuous image of a non-archemedian space such that each point pre-image is either a point or a compact $G_{\delta}$-set (having branches of equal length).

IV. $M_{i}$-normality. Denote by $M(X)$ the set of all ordered pairs $(H, K)$ of disjoint closed subsets of the space $X$. A function $S$ from $M(X)$ to the open subsets of $X$ is called a normality operator if for each $(H, K) \in M(X), H \subset S(H, K) \subset \mathrm{Cl} S(H, K) \subset X-K$. Clearly, $X$ is normal if and only if it admits a normality operator.

A normality operator $S$ may have the following properties:

(1) $S$ is canonical if for each $(H, K) \in M(X), S(H, K)$ is canonical;

(2) $S$ is monotone if for each $(H, K),\left(H^{\prime}, K^{\prime}\right) \in M(X), H^{\prime} \subset H$ and $K \subset K^{\prime}$ implies $S\left(H^{\prime}, K^{\prime}\right) \subset S(H, K)$;

(3) $S$ is strongly monotone if for each $(H, K),\left(H^{\prime}, K^{\prime}\right) \in M(X)$, $H^{\prime} \subset S(H, K)$ and $K \subset K^{\prime}$ implies that $S\left(H^{\prime}, K^{\prime}\right) \subset S(H, K)$;

(4) $S$ is symmetric if for each $(H, K) \in M(X), S(H, K) \cap S(K, H)$ $=0$

(5) $S$ is strongly symmetric if for each $(H, K) \in M(X), \mathrm{Cl} S(H, K) \cap$ $\mathrm{Cl} S(K, H)=0$.

A space which admits a normality operator which is:

(1) strongly monotone and canonical is said to be $M_{1}$-normal;

(2) strongly monotone is said to be $M_{2}$-normal; and

(3) monotone is said to be $M_{3}$-normal or monotonically normal.

The normality operator associated with an $M_{i}$-normal space ( $i=$ $1,2,3)$ is called an $M_{i}$-normality operator and an $M_{3}$-normality operator is also called a monotone normality operator.

The term monotonically normal was introduced by Zenor [16] and the definitions of $M_{1}$ and $M_{2}$-normality are also due to Zenor. It is shown in [4] that a montonically normal space admits a symmetric monotone normality operator.

THEOREM 7. The (irreducible) closed continuous image of a non-archemedian space admits a strongly symmetric $M_{2}\left(M_{1}\right)$-normality operator.

Proof. Let $\phi$ be an irreducible closed map from the non-archemedian space $Y$ onto the space $X$ and let $T$ be a tree whose branch space $Z$ contains $Y$.

Suppose $H$ and $K$ are disjoint closed subsets of $X$, then $\hat{\phi} H$ and $\hat{\phi K}$ are closed disjoint subsets of $Y$ although $\mathrm{Cl}_{z} \hat{\phi H}$ and $\mathrm{Cl}_{z} \hat{\phi} K$ may not be disjoint.

For each branch $z \in \hat{\phi H}$, there exists $t \in z$ such that $[t] \cap \mathrm{Cl}_{z} \hat{\phi} K=$ 0 . The collection of such $t$ which are minimal in $T$ is an anti-chain which generates a disjoint cover of $\hat{\phi H}$ and is a collection of closed-open sets. 
Denote by $\sigma(H, K)$ the largest open subset of the union of this cover such that $\hat{\phi} H \subset \sigma(H, K)$ and $\phi \sigma(H, K)$ is open in $X . \sigma(K, H)$ denotes a similar set for $\phi K$. The basis $\mathcal{E}[t](t \in T)$ is a rank-1 basis and as a consequence we have $\sigma(H, K) \cap \sigma(K, H)=0$. Clearly, $\phi \sigma$ is a normality operator for $X$ and we show the following:

\section{(A) $\phi \sigma$ is canonical.}

Since $\phi \sigma(H, K)$ is open, it is a subset of the closure of its interior. Assume the existence of $x \in \operatorname{In} C l \phi \sigma(H, K)-\phi \sigma(H, K)$, then each open set containing $x$ in its closure must intersect $\phi \sigma(H, K) . \hat{\phi(x)}$ is not covered by the collection from which we generated $\sigma(H, K)$, since if it were, then we would have $x \in \phi \sigma(H, K)$, thus there exists $t \in T$ such that $[t]$ intersects no element of this collection and $[t] \cap \hat{\phi(x)} \neq 0$. In case $\phi$ is irreducible, $\phi[t]$ has a non-empty interior with $x$ a boundary point, but In $\phi[t] \cap \phi \sigma(H, K)=0$, a contradiction. Notice that this is the only use made of irreducibility in this proof. In the absence of irreducibility, we obtain $M_{2}$ instead of $M_{1}$-normality.

(B) $\phi \sigma$ is strongly monotone.

Suppose $(H, K),\left(H^{\prime}, K^{\prime}\right) \in M(X)$ with $H^{\prime} \subset \phi \sigma(H, K)$ and $K \subset K^{\prime}$. Note that $\hat{\phi} H^{\prime} \subset \sigma(H, K)$ since if not then some point of $H^{\prime}$ must be in the boundary of $\phi \sigma(H, K)$.

The facts that $Z$ has a rank-1 base and that $K \subset K^{\prime}$ together imply that the cover of $\hat{\phi} H^{\prime}$ from which $\sigma\left(H^{\prime}, K^{\prime}\right)$ is constructed is a refinement of the cover from which $\sigma(H, K)$ was constructed, since these covers use minimal elements of $T$. It follows that $\sigma\left(H^{\prime}, K^{\prime}\right) \subset \sigma(H, K)$, so we have $\phi \sigma\left(H^{\prime}, K^{\prime}\right) \subset \phi \sigma(H, K)$.

We have now shown that $\phi \sigma$ is a symmetric $M_{2}\left(M_{1}\right)$-normality operator, unfortunately $\phi \sigma$ is not necessarily strongly symmetric. We will now construct from $\phi \sigma$ an $M_{2}\left(M_{1}\right)$-normality operator which is strongly symmetric.

Let $S(H, K)=\phi \sigma(H, \mathrm{Cl} \phi \sigma(K, H))$. Clearly, $S$ is a (canoncial and) strongly symmetric normality operator and we must now show that it strongly monotone.

Suppose $H^{\prime} \subset S(H, K)$ and $K \subset K^{\prime}$. Notice that $S\left(H^{\prime}, K^{\prime}\right)$ is obtained by using $\phi \sigma$ to separate $H^{\prime}$ from $\mathrm{Cl} \phi \sigma\left(K^{\prime}, H^{\prime}\right)$ and that we will then have $S\left(H^{\prime}, K^{\prime}\right) \subset S(H, K)$, the strong monotone property, whenever it holds that $\phi \sigma(K, H) \subset \phi \sigma\left(K^{\prime}, H^{\prime}\right)$.

We will now show that this does indeed hold. Recall that we obtained $\sigma(K, H)$ from a cover of $\hat{\phi} K$ and this cover was constructed from the minimal elements $t \in T$ such that $[t] \cap \mathrm{Cl}_{z} \hat{\phi H}=0$. Suppose $t$ is used to 
construct the cover for $\hat{\phi K}$. Since $K \subset K^{\prime}$ and $\hat{\phi} H^{\prime} \subset \sigma(H, K)$, it follows that $[t]$ has the property that it intersects $K^{\prime}$ and that it does not intersect $\mathrm{Cl}_{z} \hat{\phi} H^{\prime}$, thus $t$ or some element of $T$ which precedes $t$ will be used in the construction of the cover of $K^{\prime}$ from which we obtain $\sigma\left(K^{\prime}, H^{\prime}\right)$. This implies that $\sigma(K, H) \subset \sigma\left(K^{\prime}, H^{\prime}\right)$ and thus $\phi \sigma(K, H) \subset \phi \sigma\left(K^{\prime}, H^{\prime}\right)$.

COROLLARY 1. Each proto-metrizable space admits a strongly symmetric $M_{1}$-normality operator.

Lasnev [7] has investigated the closed continuous images of metrizable spaces and such spaces now bear his name. Using [7, Theorem 4], each Lasnev space is the irreducible closed continuous image of some metrizable non-archemedian space and we have:

COROLlaRy 2. Each Lasnev space admits a strongly symmetric $M_{1^{-}}$ normality operator.

V. Examples. We have previously characterized a proto-metrizable space as the image of a non-archemedian space under a closed map having the property that each point pre-image is either a point or a compact $G_{\delta}$-set. This property can be weakened to obtain the following classes of space:

(1) The class $\mathcal{K}$ of spaces which are the image of a non-archemedian space under a closed map such that point pre-images are compact. (The perfect images of non-archemedian spaces.)

(2) The class $\mathcal{G}$ of spaces which are the image of a non-archemedian space under a closed map such that each point pre-image is a monotone $G_{\delta}(\alpha)$-set for some $\alpha$.

(3) The class $\mathcal{C}$ of spaces which are the closed continuous image of a non-archemedian space.

It is the purpose of this section to give examples showing that these classes of spaces are disjoint from the class of proto-metrizable spaces and from each other.

First we establish that the class of proto-metrizable spaces coincides with the intersection of $\mathscr{K}$ and $\mathcal{G}$. If $X$ is a member of $\mathcal{G}$, then each point pre-image is a monotone $G_{\delta}(\alpha)$-set for some $\alpha$. If $X$ is also a member of $\mathscr{K}$, that is, if $X$ can be represented as the perfect image of a non-archemedian space, then the inverse of this map preserves monotone $G_{\delta}(\alpha)$-sets and thus by Theorem $6(\mathrm{f}), X$ is proto-metrizable.

EXAMPLE 1 . Let $T$ be the tree having exactly two branches, $b(0)$ of length $\omega$ and $b(1)$ of length $\omega(1)$ and let $\phi$ be the map which identifies 
these two branches so that $X=\{x\}=\{\phi(b(0))\}=\{\phi(b(1))\}$. Let $T^{\prime}$ and $\phi^{\prime}$ be the tree and map constructed from $T$ and $\phi$ using the techniques of Lemma 5.

The image $X^{\prime}$ of the branch space of $T^{\prime}$ under the map $\phi^{\prime}$ clearly belongs to the class $\mathscr{K}$ and we show that $X^{\prime}$ does not have an ortho-base, hence is not proto-metrizable and thus cannot belong to $\mathcal{G}$.

We choose from a basis for $X^{\prime}$ a countable perfectly decreasing subcollection such that $x$ is an element of its intersection and each element contains a branch with endpoint in $b(0) \times\{1\}$ which the next element does not contain. Since no countable set is cofinal in $\omega(1)$, the intersection of this collection must have non-empty interior, but the intersection is not open since it contains $x$ as a boundary point. It follows that $X^{\prime}$ has no ortho-base.

ExAmple 2. Let $T$ be the disjoint union of $\kappa$ many Cantor trees (each branch has length $\omega$ and each level is finite), where $\kappa$ is an infinite cardinal. Let $H=\varepsilon h(\alpha)(\alpha \in \kappa)$ be a set of branches, one from each of the Cantor trees, so that $H$ is a discrete set of cardinality $\kappa$.

Let $\phi$ be the map such that $H$ is the pre-image of the point $x$ in the image space $X$ and is the identity elsewhere. Clearly $X$ is a member of the class $\mathcal{G}$.

We show that $X$ is not a member of $\mathscr{K}$ by showing that it does not have an ortho-base, hence is not proto-metrizable. Assume that $X$ has an ortho-base $B$ and then, since $\{x\}$ is a $G_{\delta}$-set, we construct a countable subcollection $B^{\prime}=\mathscr{E} b(n)(n \in \omega)$ of $B$ such that for each $n \in \omega$, $b(n+1) \subset b(n)$ and $\cap B^{\prime}=\{x\}$.

For each $\alpha \in \kappa$, let $t(\alpha)$ be the minimal element of $h(\alpha)$ such that $\phi[t(\alpha)] \subset b(n)$, where $\alpha=\alpha^{\prime}+n, \alpha^{\prime}$ is a limit ordinal and $n \in \omega$. Our assumption that $B$ is an ortho-base implies that $B^{\prime}$ must contain a base at $x$, but this is not the case, since In $\mathrm{Cl} \cup \phi[t(\alpha)](\alpha \in \kappa)$ is an open set containing $x$ but containing no element of $B^{\prime}$ as a subset.

EXAMPLE 3. Let $T_{2}$ and $H_{2}$ be the tree and closed set constructed in Example 2 and let $T_{1}$ be the sub-tree of the tree in Example 1 consisting only of all branches of length greater that $\omega$. Let $T$ be the disjoint union of these trees, let $\mathrm{H}$ consist of $\mathrm{H}_{2}$ and the $\omega(1)$-branch, and let $\phi$ be the map defined on the branch space of $T$ whose image $X$ contains $\{x\}=\phi H$.

Denote by $X_{1}$ and $X_{2}$ respectively the images under $\phi$ of the branch spaces of $T_{1}$ and $T_{2}$, then $X=X_{1} \cup X_{2}$ and $\{x\}=X_{1} \cap X_{2}$.

Evidently $X$ is not in $\mathcal{G}$ and neither is $X$ in $\mathscr{K}$ since if we assume that it is, then $X$ is the perfect image of some non-archemedian space implying that $X_{2}$ is in $\mathscr{K}$ which we have already shown not to be the case. 


\section{REFERENCES}

1. P. Aleksandrov and P. Uryson, Memoire sur les espaces topologiques compactes, Verh. Ak. Wet. Amst., 14 (1962), 1-96.

2. M. M. Coban, Spaces with bases of rank one, Akad. Nauk. Molauskat SSR. Izvestija Bul. Akad. de RSS Moldovenest Vol. 3, (1973), 12-19 and 91 (Russian).

3. G. Gruenhage and P. Zenor, Proto-metrizable spaces, Houston J. Math., 3 (1977).

4. R. W. Heath, D. J. Lutzer and P. L. Zenor, Monotonically normal spaces, Trans. Amer. Math. Soc., 178 (1973), 481-493.

5. T. J. Jech, Lectures in set theory, Lecture Notes in Mathematics, vol. 217, SpringerVerlag, New York, 1971.

6. I. Juhasz, Cardinal functions in topology, Mathematical Center Tract, vol. 34, Matematische Centrum, Amsterdam, 1971.

7. N. S. Lasnev, Continuous decomposition and closed mappings of metric spaces, Sov. Math. Dokl., 6 (1965), 1504-1506.

8. E. Michael, Another note on paracompact spaces, Proc. Amer. Math., 8 (1957), $822-828$.

9. J. Nagata, Modern general topology, 2nd ed., Bibliotheca Mathematica, vol. 7, American Elsevier, New York, 1974.

10. P. J. Nyikos, A survey of zero dimensional spaces, Topology Proceedings of the Memphis State Univ. Conference, Franklin and Thomas, ed., Lecture Notes in Pure and Applied Mathematics, vol. 24, Marcel Dekker, New York, (1977), 87-114.

11. _ Base conditions, generalized metric spaces and metrization, (to appear).

12. __ Some surprising base properties in topology, Studies in Topology, Stavrakas and Allen, ed., (Proc. Top. Conf. Univ. of North Carolina at Charlotte), Academic Press, $1975,427-450$.

13. S Some surprising base properties in topology II, Set-theoretic Topology, G. M. Reed, ed., Academic Press, New York, 1977, 277-305.

14. V. I. Ponomarev, Projective spectra and continuous mappings of paracompacta, Amer. Math. Soc. Transl., Series 2, 39 (1964), 133-164.

15. J. E. Vaughan, Some recent results in the theory of $[a, b]$-compactness, TOPO 72--General Topology and its Applications, Lecture Notes in Mathematics, vol. 378, SpringerVerlag, New York, 1974.

16. P. Zenor, Monotonically normal spaces, Notices Amer. Math. Soc., 17 (1970), 1034, Abstract \#679-G2.

Received February 14, 1978 and in revised form June 26, 1980. This paper is the author's doctoral dissertation at Auburn University prepared under the supervision of Professor Phillip Zenor.

AUBURN UNIVERSITY

AUBURN, Al 36830 



\section{PACIFIC JOURNAL OF MATHEMATICS \\ EDITORS}

DONALD BABBITT (Managing Editor)

University of California

Los Angeles, CA 90024

\section{Hugo Rossi}

University of Utah

Salt Lake City, UT 84112

C. C. Moore and Arthur Ogus

University of California

Berkeley, CA 94720
J. DugundiI

Department of Mathematics

University of Southern California

Los Angeles, CA 90089-1113

R. FINN and H. SAMELSON

Stanford University

Stanford, CA 94305

ASSOCIATE EDITORS
R. ARens
E. F. BECKENBACH
B. H. NeumanN
F. WOLF
K. YoshidA (1906-1982)

\section{SUPPORTING INSTITUTIONS}

UNIVERSITY OF ARIZONA

UNIVERSITY OF BRITISH COLUMBIA

CALIFORNIA INSTITUTE OF TECHNOLOGY

UNIVERSITY OF CALIFORNIA

MONTANA STATE UNIVERSITY

UNIVERSITY OF NEVADA, RENO

NEW MEXICO STATE UNIVERSITY

OREGON STATE UNIVERSITY
UNIVERSITY OF OREGON

UNIVERSITY OF SOUTHERN CALIFORNIA

STANFORD UNIVERSITY

UNIVERSITY OF HAWAII

UNIVERSITY OF TOKYO

UNIVERSITY OF UTAH

WASHINGTON STATE UNIVERSITY

UNIVERSITY OF WASHINGTON 


\section{Pacific Journal of Mathematics}

Vol. 104, No. 1

May, 1983

Nestor Edgardo Aguilera and Eleonor Ofelia Harboure de Aguilera, On

the search for weighted norm inequalities for the Fourier transform $\ldots \ldots .1$

Jin Akiyama, Frank Harary and Phillip Arthur Ostrand, A graph and its complement with specified properties. VI. Chromatic and achromatic numbers ......................................... 15

Bing Ren Li, The perturbation theory for linear operators of discrete type . . . 29

Peter Botta, Stephen J. Pierce and William E. Watkins, Linear

transformations that preserve the nilpotent matrices .............. 39

Frederick Ronald Cohen, Ralph Cohen, Nicholas J. Kuhn and Joseph

Alvin Neisendorfer, Bundles over configuration spaces .......... 47

Luther Bush Fuller, Trees and proto-metrizable spaces . . . . . . . . . . 55

Giovanni P. Galdi and Salvatore Rionero, On the best conditions on the

gradient of pressure for uniqueness of viscous flows in the whole space . . 77

John R. Graef, Limit circle type results for sublinear equations $\ldots \ldots \ldots \ldots 85$

Andrzej Granas, Ronald Bernard Guenther and John Walter Lee,

Topological transversality. II. Applications to the Neumann problem for

$y^{\prime \prime}=f\left(t, y, y^{\prime}\right) \ldots \ldots \ldots \ldots \ldots \ldots \ldots \ldots \ldots \ldots \ldots \ldots \ldots \ldots \ldots . \ldots 5$

Richard Howard Hudson and Kenneth S. Williams, Extensions of

theorems of Cunningham-Aigner and Hasse-Evans . . . . . . . . . . . 111

John Francis Kurtzke, Jr., Centralizers of irregular elements in reductive algebraic groups

James F. Lawrence, Lopsided sets and orthant-intersection by convex

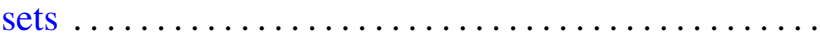

Åsvald Lima, G. H. Olsen and U. Uttersrud, Intersections of $M$-ideals and

$G$-spaces

Wallace Smith Martindale, III and C. Robert Miers, On the iterates of derivations of prime rings

Thomas H. Pate, Jr, A characterization of a Neuberger type iteration procedure that leads to solutions of classical boundary value problems

Carl L. Prather and Ken Shaw, Zeros of successive iterates of multiplier-sequence operators

Billy E. Rhoades, The fine spectra for weighted mean operators

Rudolf J. Taschner, A general version of van der Corput's difference theorem

Johannes A. Van Casteren, Operators similar to unitary or selfadjoint ones 